\title{
DNA sequencing of maternal plasma reliably identifies trisomy 18 and trisomy 13 as well as Down syndrome: an international collaborative study
}

\author{
Glenn E. Palomaki, PhD'1, Cosmin Deciu, MS², Edward M. Kloza, MS', \\ Geralyn M. Lambert-Messerlian, PhD'1, James E. Haddow, MD¹, Louis M. Neveux, BA', \\ Mathias Ehrich, $\mathrm{MD}^{3}$, Dirk van den Boom, $\mathrm{PhD}^{3}$, Allan T. Bombard MD, MBA ${ }^{2-4}$, \\ Wayne W. Grody, MD, PhD ${ }^{5-7}$, Stanley F. Nelson, MD ${ }^{5,7,8}$ and Jacob A. Canick, PhD ${ }^{1}$
}

Purpose: To determine whether maternal plasma cell-free DNA sequencing can effectively identify trisomy 18 and 13.

Methods: Sixty-two pregnancies with trisomy 18 and 12 with trisomy 13 were selected from a cohort of 4,664 pregnancies along with matched euploid controls (including 212 additional Down syndrome and matched controls already reported), and their samples tested using a laboratory-developed, next-generation sequencing test. Interpretation of the results for chromosome 18 and 13 included adjustment for CG content bias.

Results: Among the $99.1 \%$ of samples interpreted $(1,971 / 1,988)$, observed trisomy 18 and 13 detection rates were $100 \%(59 / 59)$ and $91.7 \%(11 / 12)$ at false-positive rates of $0.28 \%$ and $0.97 \%$, respectively. Among the 17 samples without an interpretation, three were trisomy 18. If $z$-score cutoffs for trisomy 18 and 13 were raised slightly, the overall false-positive rates for the three aneuploidies could be as low as $0.1 \%(2 / 1,688)$ at an overall detection rate of $98.9 \%(280 / 283)$ for common aneuploidies. An independent academic laboratory confirmed performance in a subset.

Conclusion: Among high-risk pregnancies, sequencing circulating cell-free DNA detects nearly all cases of Down syndrome, trisomy 18 , and trisomy 13 , at a low false-positive rate. This can potentially reduce invasive diagnostic procedures and related fetal losses by $95 \%$. Evidence supports clinical testing for these aneuploidies.

Genet Med 2012:14(3):296-305

Key Words: clinical validation; detection rate; false-positive rate; fetal DNA; massively parallel shotgun sequencing; prenatal screening; trisomy 18 ; trisomy 13

\section{INTRODUCTION}

The main focus of prenatal screening programs is to identify fetuses with Down syndrome or open neural tube defects. Identification of two less common autosomal aneuploidiestrisomy 18 (Edwards syndrome) and trisomy 13 (Patau syndrome) - is, however, an important secondary aim. In the absence of prenatal diagnosis and selective termination, an estimated 7,730 cases with Down syndrome, 1,330 with trisomy 18 , and 600 with trisomy 13 are expected at term among the 4.25 million pregnancies in the United States each year. ${ }^{1,2}$ Trisomy 18 and 13, as well as Down syndrome (trisomy 21), are more common in the first and second trimesters than at term, owing to high rates of spontaneous loss. ${ }^{3}$ Neither trisomy 18 nor trisomy 13 is associated with long-term survival, with $5-10 \%$ of live-born infants surviving $>1$ year. ${ }^{4,5}$ Most ultrasound/ biochemical screening programs for Down syndrome currently provide targeted interpretations for trisomy 18 and/or trisomy 13 that allow for a majority of these cases to be detected $(60 \%$ or higher), at low false-positive rates ( $1 \%$ or lower) ${ }^{6-8}$

Next-generation sequencing of circulating cell-free DNA in maternal plasma is capable of identifying nearly all Down syndrome pregnancies with low false-positive rates, ${ }^{9-13}$ but achieving that level of performance for identifying trisomy 18 and 13 is expected to be more difficult, owing mainly to higher variability of their percent genomic representation in euploid pregnancies. ${ }^{12-14}$ As a result, the successful application of such testing for all three of these autosomal aneuploidies has not yet been described in a clinical setting, despite a reanalysis of the initial data that demonstrated clear improvement. ${ }^{15}$ The present study addresses this issue.

\footnotetext{
${ }^{1}$ Division of Medical Screening and Special Testing, Department of Pathology and Laboratory Medicine, Women \& Infants Hospital, Alpert Medical School of Brown University, Providence, Rhode Island, USA; ${ }^{2}$ Sequenom Center for Molecular Medicine, San Diego, California, USA; ${ }^{3}$ Sequenom Inc., San Diego, California, USA; ${ }^{4}$ Department of Reproductive Medicine, University of California-San Diego, San Diego, California, USA; ${ }^{5}$ Department of Pathology and Laboratory Medicine, David Geffen School of Medicine, University of California-Los Angeles, Los Angeles, California, USA; ${ }^{6}$ Department of Pediatrics, David Geffen School of Medicine, University of California-Los Angeles, Los Angeles, California, USA; ${ }^{7}$ Department of Human Genetics, David Geffen School of Medicine, University of California-Los Angeles, Los Angeles, California, USA; ${ }^{8}$ Department of Psychiatry and Biobehavioral Sciences, David Geffen School of Medicine, University of California-Los Angeles, Los Angeles, California, USA. Correspondence: Glenn E. Palomaki (gpalomaki@ipmms.org) 


\section{MATERIALS AND METHODS}

Ensuring the study's integrity

Strategies and actions to ensure the study's integrity have been described earlier..$^{10}$ Briefly, an independent three-person oversight committee was established, an independent laboratory provided confirmatory testing, standard operating protocols were written and implemented in Clinical Laboratory Improvement Amendments-approved laboratory settings, and steps were taken to ensure the isolation of enrollment sites and outcome information from the study sponsor and testing laboratories.

\section{Sample collection}

An international collaboration of 27 prenatal diagnostic centers collected and processed the maternal plasma samples from 4,664 women before their diagnostic testing in the late first and early second trimester (ClinicalTrials.gov NCT00877292). From this cohort, a nested case/control study was designed. The results of testing 212 pregnancies with Down syndrome and their 1,484 matched controls have been published. ${ }^{10}$ During that same 9-week testing period, samples from pregnancies with trisomy 18 and 13 and their controls were also tested. Inclusion criteria were the same as for the earlier study of Down syndrome. Only samples from singleton pregnancies were included; samples with known mosaicism for trisomy 18 or 13 were excluded. Each pregnancy with trisomy 18 and 13 was matched with three controls based on the gestational age (nearest week, same trimester), enrollment site, race, and time in freezer (within 1 month). The present analysis also includes results from all Down syndrome samples and their euploid controls previously reported, with all samples being tested and interpreted for trisomy 18 and 13, as well as for Down syndrome.

\section{Massively parallel shotgun sequencing}

The laboratory-developed, plasma-based DNA test has been described. ${ }^{9,10}$ In brief, circulating cell-free DNA fragments were isolated from maternal plasma and the fetal fraction determined using a published method relying on differentially methylated markers. ${ }^{9,16}$ The remaining isolate was used to generate sequencing libraries. These were normalized and multiplexed (four samples per lane), allowing 32 samples to be run per flow cell. The flow cells were sequenced on the Illumina HiSeq 2000 (San Diego, CA), and the resulting data were analyzed using Illumina software. The laboratory-developed test for Down syndrome had been verified at the Sequenom Center for Molecular Medicine (SCMM) in San Diego before testing on this data set. ${ }^{9}$ By contrast, the test for trisomy 18 and 13 was not formally verified at SCMM before being applied to this data set, because of the limited availability of cases. However, an algorithm, which included routine GC adjustment of chromosome 18 and 13 counts, ${ }^{15,16}$ and the setting of flow-cell specific cutoff levels was developed and "locked down," before any laboratory testing. Accounting for the GC-content bias involved multiplying the raw matched reads by a correction factor derived from the relationship between GC content of each 50-kb "bin" across the genome, versus the number of matched reads. ${ }^{15,16}$ Computer interpretation relied on a robust estimate of the standard deviations above or below the central estimate $(z \text {-score })^{10}$ for each chromosome of interest $(21,18$, and 13). $z$-Scores at or above 3 were considered to be indicative of Down syndrome, trisomy 18, and trisomy 13, respectively. When evaluating the sequencing data, potential abnormalities in all three chromosomes were examined simultaneously. The euploid pregnancies were considered to be controls for each chromosome. All results were reviewed by the laboratory director, who had discretion on the final interpretation and the ability to request that a second aliquot be tested.

\section{Statistical analyses}

Three chromosome-specific detection rates and their corresponding false-positive rates were computed. An overall false-positive rate was also computed, as more than one falsepositive result might occur in a single sample. Results were also expressed as multiples of the plate-specific median (MoM) for control pregnancies. Confidence intervals were computed using the binomial distribution, using True Epistat (Richardson, TX). Rates and proportions were compared using the $t$-test, analysis of variance, $\chi 2$, or Fisher's exact test (SAS, Cary, NC). $P$ values were two-sided with significance defined at the 0.05 level.

\section{Modeling the potential impact of massively parallel shotgun sequencing testing}

The model offers diagnostic testing (amniocentesis or chorionic villus sampling) only to those women with the highest risks. It begins with routine prenatal screening tests commonly used in the United States and assesses the impact of subsequent massively parallel shotgun sequencing (MPSS) testing in those pregnancies with screen-positive results. We used the vital statistics report, indicating $\sim 4.25$ US million births in 2008 and including the distribution of maternal ages at delivery. ${ }^{2}$ As part of the College of American Pathologists 2011 FP-A Survey, ${ }^{17}$ participants were asked what type of screening test(s) they offered and how many were performed monthly (used with permission). In the United States, the 119 respondents reported 2.61 million women tested during 2010 (61\%). This is likely to be an underestimate, given that 34 participants did not respond and that some screening laboratories do not participate in the survey. A reasonable estimate is 2.8 million (two-thirds of the 4.25 million births).

Three Down syndrome screening tests were modeled: (i) combined testing (nuchal translucency ultrasound measurement and serum measurements of PAPP-A and the free $\beta$ subunit of human chorionic gonadotropin at about 11-13 weeks' gestation); (ii) quadruple testing (serum measurements of a-fetoprotein, unconjugated estriol, human chorionic gonadotropin, and inhibin-A at about 15-20 weeks' gestation); and (iii) integrated/sequential testing (information from both the combined and quadruple test together). The corresponding Down syndrome and trisomy 18 detection rates 
and false-positive rates are based on published parameters ${ }^{18}$ and the 2008 maternal age distribution. Studies have documented that the uptake of diagnostic testing is dependent on the reported risk, with higher uptake associated with higher risks, ${ }^{19-21}$ and the model uses data from one of these ${ }^{19}$ for the diagnostic testing uptake rate after a screening test, as well as after a positive MPSS test. The model relies on a summary estimate of $99 \%$ detection for Down syndrome and trisomy 18 at an overall $0.5 \%$ false-positive rate for MPSS testing, along with a $0.9 \%$ failure rate. The model outcomes include Down syndrome and trisomy 18 detection rates, numbers of women offered diagnostic testing, diagnostic procedures avoided, and procedure-related losses avoided. Each screening test is separately modeled using the US population. Trisomy 13 is not included in the model because of its lower prevalence, as well as imprecise or biased estimates for detection and falsepositive rates.

\section{RESULTS}

\section{Study samples}

Among the 4,664 samples collected, 62 samples with trisomy 18 and 12 with trisomy 13 were identified and included in the nested case/control study. Among the trisomy 18 samples, one was initially selected as a euploid control for a Down syndrome pregnancy based on the results of chorionic villus sampling, but later correctly reported to be trisomy 18 after testing the products of conception. This one sample has no matched controls. Thus, 1,988 tests performed in 286 trisomic pregnancies and 1,702 euploid pregnancies were available for analysis. These include 212 samples with Down syndrome and 1,483 matched euploid samples from the earlier study, 62 with trisomy 18 along with 183 $(61 \times 3)$ matched euploid samples, and 12 with trisomy 13 along with 36 matched euploid samples. Figure 1 summarizes these numbers, stratified by the trimester in which the sample was collected. A summary of demographic and pregnancy-related characteristics of the trisomy 18 and 13 cases, and their matched euploid pregnancies, is shown in Table 1. Overall, 110 samples failed the initial MPSS testing, 105 of which required repeat testing using a second aliquot. Five were successfully rerun without a second aliquot. A final interpretation was ultimately provided for 93 of the 110 $(84 \%)$. Among the 17 that failed, the most common reason was a fetal fraction under the prespecified lower acceptable limit of $4 \%$. Among the 17 failures were three trisomy 18 and 14 euploid pregnancies. One additional partial failure occurred in a sample with borderline quality parameters. The laboratory director correctly signed out that sample as representing Down syndrome but would not provide an interpretation for chromosome 18 or 13 . The final failure rate was $0.9 \%(17 / 1,988,95 \%$ CI $0.5-1.4 \%)$. Testing was successful in the remaining 1,971 samples, and results were formally reviewed and signed out by the laboratory director. Overall, usable test results and interpretations were achieved in 1,688 euploid and 283 trisomic pregnancies.

\section{Trisomy 18 test performance}

The three trisomy 18 failures were all due to fetal fractions of $3 \%$ or lower on both aliquots. Figure 2 a shows the chromosome $18 z$-scores versus the fetal fraction for the 59 remaining trisomy 18 samples, 1,688 euploid samples, as well as the Down syndrome and trisomy 13 samples (totaling 1,971). There was complete separation between the two groups. All 59 samples with trisomy 18 with an interpretation were associated with $z$-scores of 3.88 or higher, and were signed out as being consistent with trisomy 18 . The detection rate among the interpreted samples was $100 \%$ (59/59, 95\% CI 93.9$100 \%)$. Among the euploid samples, five had $z$-scores of 3.00 or higher, the highest being 3.46. One $z$-score from a Down syndrome pregnancy was also elevated ( $z$-score of 3.14 , see Supplementary Table S1 online), but was correctly interpreted as Down syndrome by the laboratory director. The false-positive rate for chromosome 18 was $0.3 \%(5 / 1,688$, 95\% CI $0.1-0.7 \%)$.

\section{Trisomy 13 test performance}

Figure $2 \mathbf{b}$ shows the chromosome $13 z$-scores versus the fetal fraction for the same 1,971 samples. The one false-negative was near the center of the euploid population $(z$-score of -0.19$)$, and the one false-positive was unusually high ( $z$-score of 10.94). Except for these two results, there was complete separation. Among the 12 pregnancies with trisomy 13, 11 were associated with $z$-scores above 7.17 and were signed out as being consistent with trisomy 13 . The single remaining trisomy 13 sample was signed out as normal (false-negative). The detection rate was $91.7 \%(11 / 12,95 \%$ CI 61-99\%). Sixteen euploid pregnancies had $z$-scores above 3.0, the highest being 10.94 . One sample from a Down syndrome pregnancy was also elevated ( $z$-score of 5.77, see Supplementary Table S1 online), but was correctly interpreted as Down syndrome by the laboratory director. The false-positive rate for chromosome 13 was $0.9 \%(16 / 1,688,95 \%$ CI $0.5-1.5 \%)$. All but one false-positive result fell below a $z$-score of 6.46 . Both the false-negative and false-positive results were extensively reviewed, and no errors were identified in processing, outcome, or testing.

\section{Down syndrome test performance}

Figure $2 \mathrm{c}$ shows a similar plot for chromosome $21 z$-score. We have previously shown that adjusting these results for GC content and utilizing repeat masking improves the performance of the $z$-score. Therefore, the results presented in Figure 1c, which reflect these improvements, will differ from the main results presented in our earlier article. ${ }^{10}$ Two $z$-scores from Down syndrome pregnancies are below 3. Based on these revised $z$-scores, the Down syndrome detection rate is $99.1 \%(210 / 212$, 95\% CI 96.6-99.9\%). Two other elevated results, one from a euploid pregnancy and one from a trisomy 18 pregnancy, were correctly interpreted (see Supplementary Table S1 online). The false-positive rate for chromosome 21 is $0.1 \%(1 / 1,688$, $95 \% \mathrm{CI}<0.1-0.3 \%)$, using the flow-cell-specific $z$-scores based on the repeat masked genome and with GC normalization. 


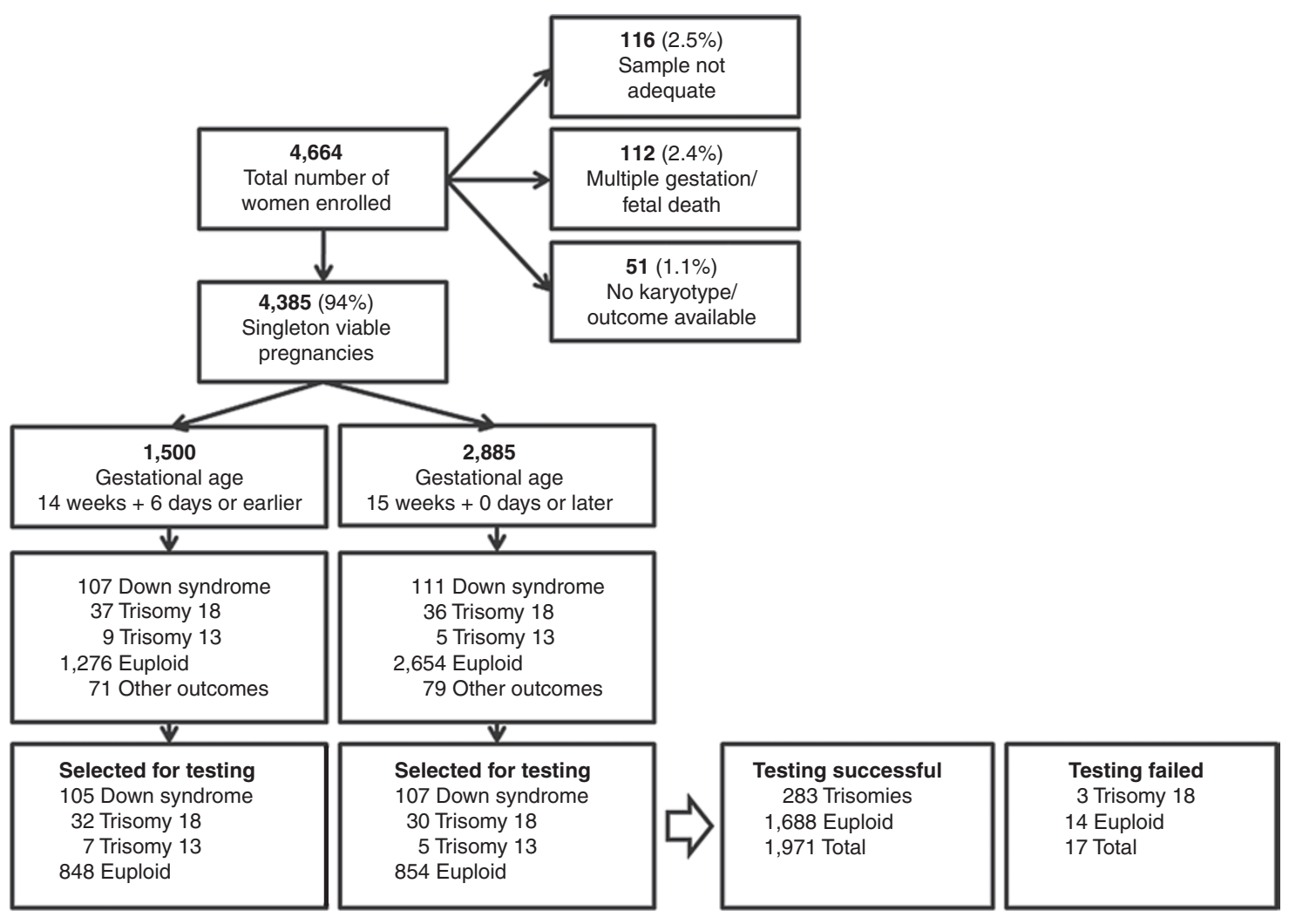

Figure 1 Flow chart showing the cohort of samples collected, those selected for testing, and the numbers for which testing was successful. This report focuses on 1,988 pregnancies subject to testing. Of these, 286 were common trisomies (Down syndrome, trisomy 18, and trisomy 13), along with 1,702 matched euploid controls.

\section{Validation by an independent academic laboratory}

The UCLA laboratory received frozen prepared library materials from SCMM for a subset of 90 samples from trisomy 18 , trisomy 13, and euploid pregnancies. They performed cluster generation, DNA sequencing, and interpretation. The laboratory director signed out individual results. The chromosome 21 results in the Down syndrome and euploid samples have already been reported. ${ }^{10}$ Of the 90 samples, 81 (90\%) were signed out by both sites. Among these results, 59 of 59 euploid, 18 of 18 trisomy 18 and four of four trisomy 13 samples were correctly classified by each laboratory (see Supplementary Figure S1 online). Among the nine initial sample failures, six were failures at both sites (four euploid and two trisomy 18 samples), usually due to low fetal fractions. Two euploid samples that failed at SCMM were successfully sequenced at UCLA and correctly classified. One additional euploid sample failed at UCLA as a result of laboratory error, but it was successfully classified by SCMM. A new aliquot was requested by SCMM for each of the eight initial failures as part of the established clinical protocol (UCLA could not make such requests); seven of these resulted in successful sequencing and correct interpretations (two trisomy 18 and five euploid pregnancies). The remaining repeat aliquot also resulted in a failure, due to the fetal fraction still being under $4 \%$.

For an additional 20 pregnancies, $4 \mathrm{ml}$ aliquots were sent to both laboratories to separately undergo complete testing. No failures occurred: three trisomy 18 , two trisomy 13 , and all 15 euploid matched samples were sequenced and correctly classified by both laboratories (see Supplementary Figure S2 online).

\section{Use of MoM rather than z-score}

MPSS results can also be expressed as MoM percent chromosome result for euploid pregnancies. ${ }^{10}$ Separate MoMs must be generated for chromosomes 21, 18, and 13. Figure 3 compares the $z$-score with MoM for correctly classifying the pregnancies and allows for selecting potentially more appropriate cutoff levels for both interpretive units. Figure 3 a compares all the results of chromosome 18, with Figure $3 \mathbf{b}$ focusing on the data near the cutoff levels. If a $z$-score cutoff between 3.4 and 3.8 were used (vertical gray rectangle), the trisomy 18 detection rate among interpreted results would be $100 \%$, with no false-positive results. A MoM cutoff level between 1.009 and 1.101 (horizontal gray rectangle) would also result in $100 \%$ detection, but with one false-positive result in a euploid pregnancy and another positive result for a Down syndrome case discussed earlier.

Figure 3c,d shows the results of chromosome 13 interpretations for the same population. If a $z$-score cutoff between 6.5 and 7.1 were used, only one false-positive in a euploid pregnancy would occur, along with one false-negative trisomy 13 case. The same performance is found for a MoM cutoff between 1.016 and 1.020 . Figure $3 e, f$ shows the results of chromosome 21 interpretations. There is no cutoff level that would clearly improve the performance over the $z$-score of 3 originally validated as part of 
Table 1 Demographic and pregnancy-related data for the trisomy 18 and 13 cases and their matched controls

\begin{tabular}{|c|c|c|c|c|}
\hline Characteristic & Trisomy 18 & Trisomy 13 & Euploid & $P$ value ${ }^{a}$ \\
\hline Number of samples & 62 & 12 & 219 & \\
\hline Maternal age in years (average, s.d.) & $36.6,4.9$ & $33.3,5.6$ & $37.6,5.0$ & 0.01 \\
\hline Gestational age (average, range, in weeks) & $14.8(10.5-21)$ & $15.2(11.4-22)$ & $14.7(9.0-21)$ & 0.83 \\
\hline Gestational age in 1st/2nd trimester (\%) & $52 / 48$ & $58 / 42$ & $52 / 48$ & 0.91 \\
\hline Maternal race $(N, \%)$ & & & & 0.95 \\
\hline Caucasian & $52(84)$ & $11(92)$ & $186(84)$ & \\
\hline Black & $3(5)$ & 0 & $9(4)$ & \\
\hline Asian & $3(5)$ & $1(8)$ & $12(6)$ & \\
\hline Main indication for enrollment $(N, \%)$ & & & & $<0.001$ \\
\hline Screen-positive by 1 st trimester test & $4(6)$ & $1(8)$ & $16(7)$ & \\
\hline Screen-positive by 2 nd trimester test & $1(2)$ & 0 & $12(5)$ & \\
\hline Screen-positive by integrated test & $7(11)$ & $1(8)$ & $22(10)$ & \\
\hline Ultrasound anomaly identified & $29(47)$ & $7(57)$ & $21(10)$ & \\
\hline Advanced maternal age & $6(10)$ & $1(8)$ & $115(53)$ & \\
\hline Two or more indications & $13(21)$ & $2(17)$ & $22(10)$ & \\
\hline Family history of aneuploidy & $2(3)$ & 0 & $8(4)$ & \\
\hline Other or not indicated & 0 & 0 & $3(1)$ & \\
\hline qfPCR or FISH alone & 0 & 0 & 0 & \\
\hline Hemolysis moderate to gross $(N, \%)$ & $1(2)$ & $1(8)$ & $6(3)$ & 0.46 \\
\hline Sample processing (avg. hours, range) & $2.0(0-5)$ & $1.8(0-4)$ & $2.0(0-6)$ & 0.99 \\
\hline
\end{tabular}

FISH, fluorescence in situ hybridization; qfPCR, quantitative fluorescence polymerase chain reaction.

aMissing values were not considered in the analysis.

the laboratory-developed test. A MoM cutoff between 1.013 and 1.016 would result in only a single false-negative, with no falsepositive results.

\section{Effect of repeat masking on trisomy 18 and 13 interpretations}

At the time the samples were originally tested, repeat masking was not available as part of the $z$-score calculations. As part of the blinded post hoc analysis for Down syndrome in our previous report, the $z$-scores were based on GC normalization and use of a repeat masked reference genome post alignment, in order to more accurately reflect the mappable genome from each chromosome. The results of trisomy 18 and 13 presented earlier were adjusted for
GC-content bias, but repeat masking was not done. We compared those results with the effect of repeat masking followed by GC correction on the results of chromosome 18 and 13 . For the results of chromosome 18, the detection rate for trisomy 18 was unchanged, but the number of euploid pregnancies with a $z$-score of 3 or higher increased from 5 to 11 . In addition, the complete separation between trisomy 18 and euploid measurements achievable with the original measurements was not maintained after repeat masking (see Supplementary Figure S3 online). For the results of chromosome 13 interpretations for, there was little impact of repeat masking on the detection rate of trisomy 13, false-positive rate, or separation between measurements from the trisomy 13 and euploid pregnancies (see Supplementary Figure S4 online). 
a

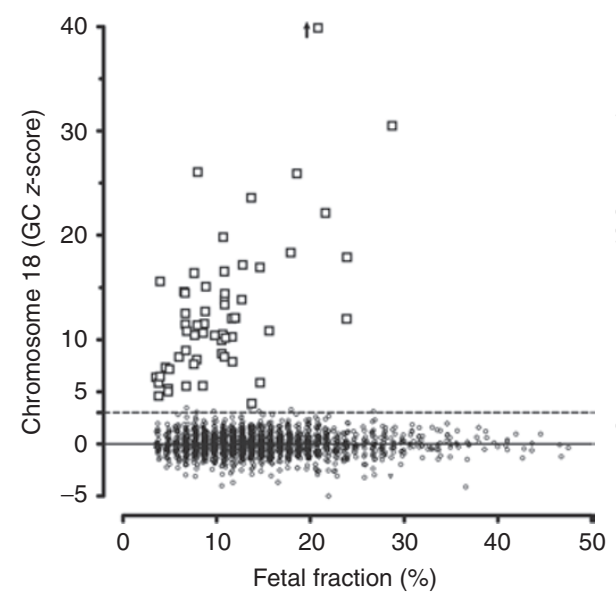

b

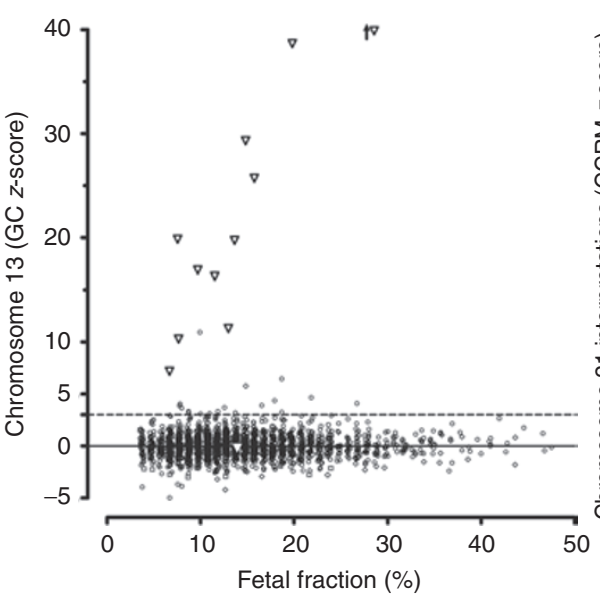

C

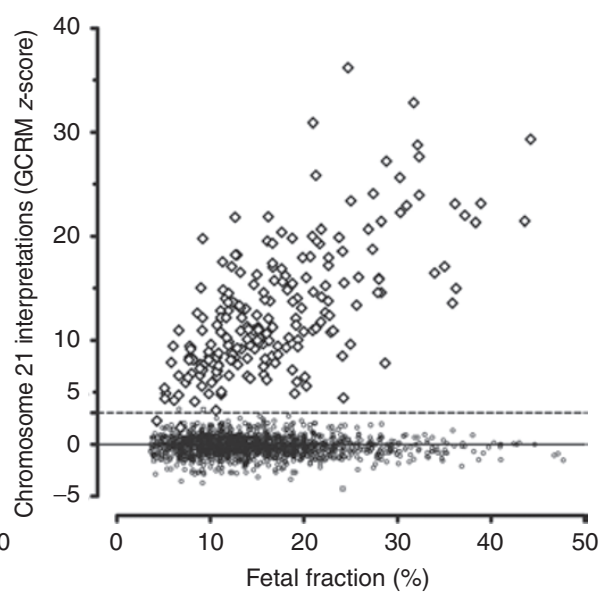

Figure 2. $\mathbf{z}$-Scores for chromosomes 18, 13, and 21. (a) Chromosome 18 -scores for 59 pregnancies with trisomy 18 and for pregnancies without trisomy 18 versus fetal fraction. Large squares indicate a trisomy 18 pregnancy. Small circles, diamonds, and triangles indicate euploid, Down syndrome, and trisomy 13 pregnancies, respectively. One $z$-score above 40 was truncated to 39.9 for display. (b) Chromosome $13 z$-scores for 12 pregnancies with trisomy 13 and for pregnancies without trisomy 13 versus fetal fraction. Large triangles indicate a trisomy 13 pregnancy. Small circles, diamonds, and squares indicate euploid, Down syndrome, and trisomy 18 pregnancies, respectively. One z-score above 40 was truncated to 39.9 for display. Note the false-negative result at a $z$-score of 0 and fetal fraction of 13\%. (c) Chromosome 21 interpretations $z$-scores for 212 Down syndrome pregnancies and for pregnancies without Down syndrome versus fetal fraction. Large diamonds indicate a Down syndrome pregnancy. Small circles, squares, and triangles indicate euploid, trisomy 18 , and trisomy 13 pregnancies, respectively.

\section{Overall MPSS test performance for detecting trisomies}

In this study, 1,971 of 1,988 samples (99.1\%) received a clinical interpretation for all three chromosomes based on a consistent $z$-score cutoff of 3.0. Of the 17 samples in which it was not possible to provide an interpretation, three occurred in trisomy 18 pregnancies and 14 in euploid pregnancies. The rate of chromosome abnormality among the failures was $18 \%$ (95\% CI 4-43\%), not significantly different from the overall rate in the study population of $14 \%([212+62+12] / 1,988,95 \%$ CI $12-16 \%)$. Among the 283 interpreted samples with one of the three common autosomal trisomies, the detection rate was $98.9 \%$ (280/283, 95\% CI 96.9-99.8\%). Two false-negatives occurred among the 212 Down syndrome pregnancies, and the third false-negative was among the 12 trisomy 13 pregnancies. The corresponding false-positive rate was $1.4 \%(24 / 1,688,95 \%$ CI $0.9-2.1 \%)$, mostly due to chromosome 18 and 13. More detailed information is available online (see Supplementary Tables S2 and S3 online). Were the $z$-score cutoff levels for trisomy 18 and 13 reset to be more restrictive, within the vertical gray zones depicted in Figure $3 b$,d, the overall detection rate could remain at $98.9 \%$, but the false-positive rate would be reduced to $0.1 \%(2 / 1,688$, $95 \% \mathrm{CI}<0.1-0.4 \%)$. Given the data-dependent nature of this estimate, a more conservative estimate of $0.5 \%$ for this lower limit of the false-positive rate is used for modeling.

\section{MPSS as a secondary test among high-risk patients}

Table 2 (additional information in Supplementary Tables S4 and 55 online) shows the impact of introducing reflexive MPSS testing for women classified as screen-positive by the combined, quadruple, or integrated/sequential screening test. The first row in Table 2 shows the performance of the combined screening test for identifying Down syndrome and trisomy 18 , followed by diagnostic testing in the women with the highest risk. Among the 2.8 million screened women, 5,156 pregnancies with Down syndrome and 888 with trisomy 18 are expected at term. The combined test identifies 4,362 cases of Down syndrome (85\%) and 772 of trisomy 18 (87\%), along with 148,079 (5.3\%) unaffected pregnancies (5\% positive for Down syndrome and $0.3 \%$ positive for trisomy 18). Together, these 153,213 women have a 1:29 odds of delivering an offspring with Down syndrome or trisomy 18. An estimated 114,307 of these women accept diagnostic testing, with uptake based on their assigned risks. An estimated 548 procedure-related losses are expected, assuming a rate of 1 loss per 200 procedures. ${ }^{22,23}$ The next two rows show the same analysis for quadruple and integrated testing.

The lower half of Table 2 shows the impact of MPSS testing on all screen-positive women before diagnostic testing. Using the combined test as an example, only $298(0.2 \%)$ of the 108,418 women with false-positive screening results are expected to also have a false-positive MPSS test. Among the 4,384 screenpositive Down syndrome pregnancies, 4,340 (98.9\%) will have a positive MPSS test. Among all women with a positive test, the odds of having a Down syndrome or trisomy 18 pregnancy is about $7: 1$. In addition, the $1,386(0.9 \%)$ with a failed test will also be offered diagnostic testing, yielding a total of 6,792 procedures and 34 procedure-related losses. The improvement gained by conducting the MPSS testing after a screen-positive result, rather than directly offering diagnostic testing is summarized in Table 2. The proportion of all prenatally diagnosed Down syndrome pregnancies improves from $78 \%$ to $84 \%$ with the use of MPSS as an intervening test; a smaller improvement is found for trisomy 18. Such use of MPSS testing also has 
a

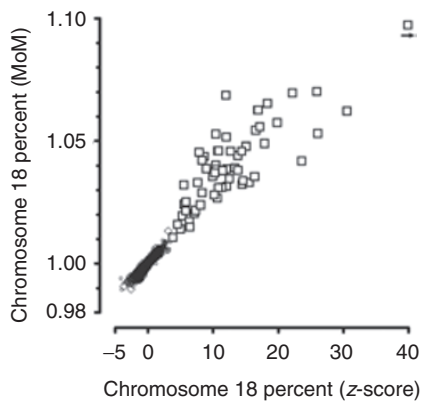

C
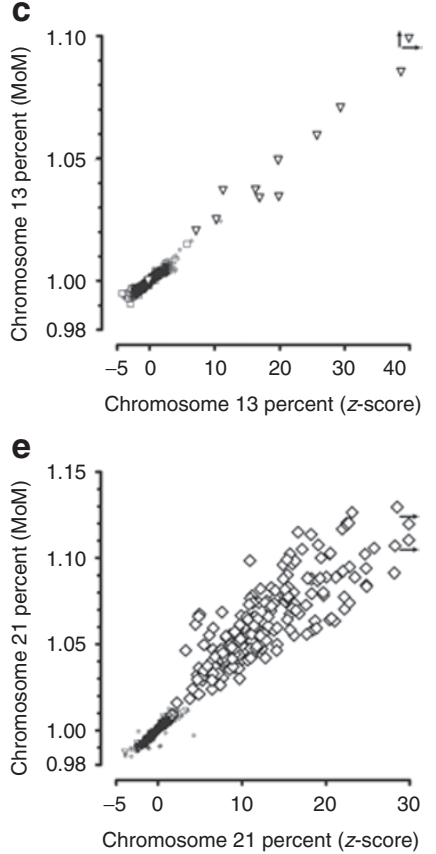

b

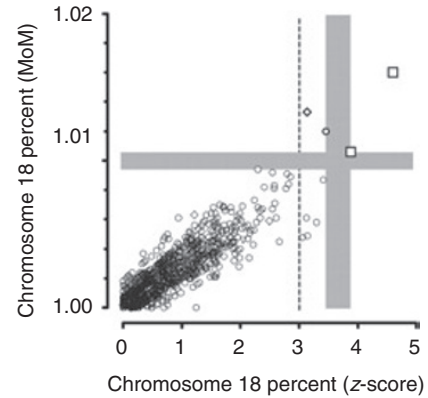

d

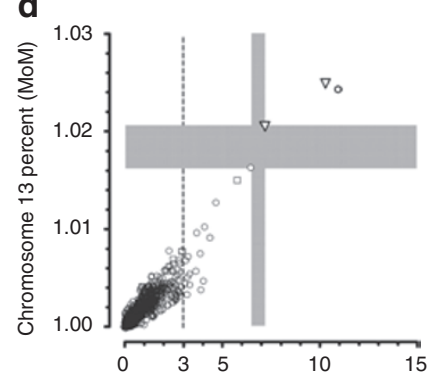

Chromosome 13 percent (z-score)

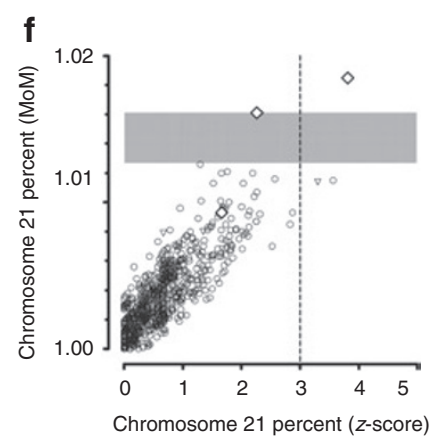

Figure 3 Interpreting chromosome 18, 13, and 21 sequencing results as $z$-scores versus multiples of the median (MoM): comparing and exploring appropriate cutoff levels. This figure shows the chromosomespecific $z$-score results are on the $x$-axis, with the same data expressed as multiples of the plate-specific median level on the $y$-axis. Results for euploid, trisomy 18 , trisomy 13 , and Down syndrome pregnancies are shown as small circles, squares, triangles, and diamonds, respectively. The aneuploidy of interest (e.g., trisomy 18 for chromosome 18 results) is shown with a larger symbol size. The top row of (a) and (b) shows the chromosome 18 results, while $(\mathbf{c})$ and (d) and (e) and (f) show results for chromosome 13 and 21, respectively. The figures on the right hand side show the same data but focus on the results near the cutoff levels. The vertical dashed line shows the original cutoff levels chosen to validate the laboratory-developed test. The gray regions show where cutoff levels might provide better screening performance in the future.

the potential to reduce procedure-related losses in the United States by $95 \%$ (from 551 to 34/year). The final two rows provide similar information for those same women tested via quadruple or integrated/sequential testing.

\section{DISCUSSION}

Together with our previous results that focused on Down syndrome,$^{10}$ this study provides strong evidence that MPSS secondary screening using maternal plasma samples from high-risk pregnancies will simultaneously identify nearly all cases of trisomy 18 and 13. An interpretation of the MPSS test for the three aneuploidies was possible for $99.1 \%$ of samples, with an overall detection rate of $98.9 \%$ (280/283). The three false-negatives include two Down syndrome and one trisomy 13 pregnancy. The corresponding false-positive rate was $1.4 \%$, but most of these could be avoided by slightly raising the $z$-score cutoff levels for trisomy 18 and 13 . The laboratorydeveloped test for these disorders had not yet been subjected to a separate in-house clinical validation when these samples were tested. Thus, the $z$-score cutoff of 3 was chosen for this study to be consistent with that found suitable for Down syndrome. Using slightly higher cutoff levels for trisomy 18 and 13 , and routinely applying GC adjustment and repeat masking for chromosome 21 interpretations would be expected to reduce the overall false-positive rate to below $0.5 \%$. However, repeat masking within the data processing for chromosome 18 and 13 interpretations provided mixed results. There was little impact on chromosome 13, but repeat masking for the result of chromosome 18 increased the false-positive rate, and resulted in more overlap between the trisomy 18 and euploid population.

A full clinical interpretation was not possible for 17 of the pregnancies, even after testing a second aliquot. Among these, the risk of aneuploidy was similar to the $15 \%$ occurring in the population with successful testing. Even in routine clinical practice, the prior risk would be sufficiently high (3-5\%, Table 2) that women with failed MPSS testing would still be offered invasive testing and chromosome analysis. Due to this opportunity for accurate diagnosis for women with a failed MPSS test, those samples were not included in the calculation of MPSS test performance.

Two previous studies have examined MPSS testing for trisomy 18 and 13. In a proof-of-concept study by Fan and colleagues, ${ }^{13} 9$ cases of trisomy 21,2 cases of trisomy 18 , and 1 case of trisomy 13 were successfully identified with no false-positive results among six euploid pregnancies. In a larger, more recent study, MPSS testing was performed on 392 maternal plasma samples, including samples from 37 pregnancies with trisomy 18 and 25 with trisomy $13 .{ }^{15}$ Using a previously standardized $z$-score method developed for trisomy 21, 27 cases of trisomy 18 cases $(73 \%)$ and 9 cases of trisomy 13 (36\%) were identified, with false-positive rates of $2.8 \%$ and $7.6 \%$, respectively. After adjustments (removal of repeat masking and accounting for GC-content bias), the detection rate for trisomy 18 and 13 improved to $91.9 \%$ at a $2.0 \%$ false-positive rate and to $100 \%$ at a $1.1 \%$ false-positive rate, respectively. This study also used GC-adjusted chromosome 18 and 13 results without repeat masking and found considerably better performance than the earlier study. ${ }^{15}$ This is most likely due to higher quality sequence data and higher total reads per patient (DNA fragments sequenced for which their chromosome of origin could be reliably identified) in this study (average 19 million without repeat masking) using the Illumina HiSeq 2000 platform, compared with those achieved using the Illumina GAIIx platform (average 4.6 million reads without repeat masking). Overall, 
Table 2 Screening and diagnostic testing of 2,800,000 women in the United States, with and without reflexive MPSS testing

\begin{tabular}{|c|c|c|c|c|c|c|c|c|c|c|c|c|}
\hline \multirow{2}{*}{ Screening test } & \multicolumn{7}{|c|}{ Screen-positive } & \multicolumn{5}{|c|}{ Directly offer Dx testing (A) } \\
\hline & \multicolumn{3}{|c|}{ DS } & T18 & \multicolumn{2}{|c|}{ All } & OAPR & UA & DS & T18 & Dx test & Fetal loss \\
\hline Combined & 148,820 & \multicolumn{2}{|c|}{4,384} & 772 & \multicolumn{2}{|c|}{153,213} & $1: 29$ & 109,578 & 3,996 & 733 & 114,307 & 548 \\
\hline Quadruple & 148,820 & \multicolumn{2}{|c|}{4,296} & 701 & \multicolumn{2}{|c|}{153,054} & $1: 30$ & 111,355 & 3,936 & 666 & 115,957 & 557 \\
\hline \multirow[t]{3}{*}{ Int/seq } & 92,661 & & & 834 & 97, & & $1: 17$ & 69,980 & 4,450 & 792 & 75,222 & 350 \\
\hline & \multicolumn{7}{|c|}{ MPSS positive then offer Dx testing (B) } & \multicolumn{5}{|c|}{ Improvements from A to B } \\
\hline & UA & DS & T18 & OAPR & Failed & Dx test & Fetal Loss & \multicolumn{2}{|c|}{ DS detection } & \multicolumn{2}{|c|}{ T18 detection } & Fetal loss \\
\hline Combined & 740 & 4,318 & 764 & $7: 1$ & 1,379 & 7,201 & 36 & $+6 \%$ & $(78,84)$ & $+3 \%$ & $(83,86)$ & $-93 \%$ \\
\hline Quadruple & 740 & 4,231 & 694 & $7: 1$ & 1,377 & 7,042 & 35 & $+6 \%$ & $(76,82)$ & $+3 \%$ & $(75,78)$ & $-94 \%$ \\
\hline Int/seq & 461 & 4,702 & 826 & $12: 1$ & 880 & 6,869 & 34 & $+5 \%$ & $(86,91)$ & $+4 \%$ & $(89,93)$ & $-90 \%$ \\
\hline
\end{tabular}

DS, Down syndrome; Dx, diagnostic (i.e., amniocentesis/chorionic villus sampling); failed, no clinical interpretation possible due to test failures on one or more quality parameters; Int/Seq, integrated/sequential; OAPR, odds of being affected given a positive result; T18, trisomy 18; UA, unaffected.

we found the addition of repeat masking to the chromosome 18 and 13 analysis to be detrimental to test performance.

The turnaround time for MPSS testing and laboratory director's sign-out of individual results has already been documented to be no more than 10 days, $90 \%$ of the time. ${ }^{10}$ The clinical advantages of offering MPSS testing as the next step after a positive screening test are substantial. Far fewer of the false-positive high-risk women will remain in that category after MPSS testing. As a result, the risk of losing an unaffected pregnancy due to an unnecessary invasive diagnostic test is expected to be substantially lower, albeit with a rare potential for a missed aneuploidy. On the other hand, a higher proportion of women who are at high risk and then receive an MPSSpositive result might opt for invasive testing. Counseling for couples with a positive MPSS test result would include information that the chance of an affected pregnancy is very high, $>50 \%$ (Table 2). The end result is the prospect that a higher proportion of women at high risk for Down syndrome, trisomy 18 , and 13 pregnancies would choose prenatal diagnosis, due to greater confidence in the screening and diagnostic process.

Currently, there is insufficient experience with MPSS testing to consider offering it as a primary screening test in the general population. In addition, the resources needed to offer it to two or three million pregnant women each year are substantial. However, introduction of MPSS testing as part of routine clinical practice can occur by offering high-risk women counseling about the benefits and risks associated with choosing MPSS testing as a secondary screening test, versus a direct offer of invasive diagnostic testing. Given the inherent delay, this decision-making process needs to be studied. Alternatively, a woman choosing serum/ultrasound based screening could provide two additional plasma samples at the outset. The plasma samples could be processed, kept in storage, and then be reflexively tested in the event that her screening result indicates high risk. This approach would reduce delays and anxiety associated with informing these women of their high-risk status in order to obtain the plasma samples for MPSS testing. It would also streamline the transition from screening to diagnostic testing in a seamless and unobtrusive manner. The relative cost of drawing and saving additional blood tubes (most of which would never be needed clinically) is a potential drawback to the reflexive testing strategy, although unused aliquots could be used for quality control and process improvements.

The detection of Down syndrome remains the primary aim of prenatal screening. However, first and second trimester interpretations for trisomy 18 and first trimester interpretations for trisomy 13 are already part of current prenatal screening practice. It is, therefore, reasonable for MPSS to have the capacity to identify these three common autosomal aneuploidies. This report demonstrates that MPSS can identify nearly all cases of Down syndrome, trisomy 18, and trisomy 13 among women at high risk for these disorders as early as 10 weeks' gestation, and can be implemented effectively in a high-risk clinical setting.

\section{SUPPLEMENTARY MATERIAL}

Supplementary material is linked to the online version of the paper at http://www.nature.com/gim

\section{ACKNOWLEDGMENTS}

We thank the oversight committee members Michael T. Mennuti (Chair), University of Pennsylvania Medical Center, Philadelphia, Pennsylvania; George A. Macones, Washington University School of Medicine, St Louis, Missouri; Iris Schrijver, Stanford University and Stanford School of Medicine, Stanford, California; and Wayne W. Grody, David Geffen School of Medicine at the University of California-Los Angeles, California, for their independent assessment of the study design and conduct, and their thoughtful advice throughout the planning and active enrollment phases.

We also thank the enrollment site personnel for their participation in the study, including North York General Hospital, Genetics Program, Toronto, Ontario, Canada: Wendy S. Meschino and Clare Gibbons; Istituto G. Gaslini, Genova, Italy: Pierangela De Biasio, Lucia Vaccari, Sabrina Bertoldi, Daniela Pastorino, Margherita Giordano, Gianluca Caridi, and Monica Dagnino; Hospital Clinic Barcelona, Barcelona, Catalonia, Spain: Antoni 


\section{ORIGINAL RESEARCH ARTICLE}

Borrell, Agusti Seres, Roser Febrero, Iratxe Torre, and Maribel Grande; Centrum Lekarske Genetiky, Ceske Budejovice, Czech Republic: David Cutka and Karel Cutka; Hospital Italiano, Buenos Aires, Argentina: Lucas Otaño, Gustavo Izbizky, and Carla Sesarini; IWK Health Center, Dalhousie University, Halifax, Canada: Michiel Van den Hof, Jo-Ann Brock, and Cora Fanning; Royal College of Surgeons in Ireland, Rotunda Hospital, Dublin, Ireland: Fergal Malone, Etaoin Kent, Julia Unterscheider, and Grainne McSorley; First Department of Ob-Gyn, Semmelweis University, Budapest, Hungary: Csaba Papp and Balint Nagy; IMALAB s.r.o. Medical Laboratories and Prediko s.r.o. Genetic and Prenatal Screening Centre, Zlin, Czech Republic: Jaroslav Loucky and Petr Polak; Genetics Section, Centro de Educacion Medica e Investigaciones Clinicas (CEMIC), Buenos Aires, Argentina: Laura Igarzabal and Florencia Petracchi; The University of lowa Hospitals and Clinics, Department of Obstetrics and Gynecology, lowa City, lowa: Kristi Borowski, Roger Williamson, Diedre Fleener, and Bruce Bedell; Prenatal Diagnosis Center, Women \& Infants Hospital, Providence, Rhode Island: Barbara M O'Brien and Jacquelyn Halliday; University of Pécs, Clinical Center Department of Obstetrics and Gynecology, Pécs, Hungary: Béla Veszprémi and Gabor Molnar; The Center for Women's Reproductive Health at the University of Alabama at Birmingham, Birmingham, Alabama: Joseph Biggio and Rachel Copper; Rambam Medical Center, Haifa, Israel: Zeev Weiner; Cedars-Sinai Prenatal Diagnostic Center, Division of Maternal-Fetal Medicine, Los Angeles, California: John Williams, Deebra Smith, and Rennatha Edwards; Northwestern University Feinberg School of Medicine, Chicago, Illinois: Jeffrey Dungan, William Grobman, Lee P. Shulman, and Suzanne Banuvar; Henry Ford Hospital, Department of Genetics, Detroit, Michigan: Jacquelyn Roberson and Ellen Martindell; University of Virginia, Department of Obstetrics and Gynecology, Charlottesville, Virginia: Devereux N. Saller Jr and Karen A. Ventura; Children's and Women's Health Centre of British Columbia, Vancouver, British Columbia, Canada: Sylvie Langlois and Patricia Power; Intermountain Healthcare and ARUP Laboratories, Salt Lake City, Utah: Nancy Rose, Elaine Lyon, and Danielle LaGrave; Brigham and Women's Hospital, Boston, Massachusetts: Louise WilkinsHaug and Diane Ahern; Baylor College of Medicine, Department of Obstetrics and Gynecology, Houston, Texas: Anthony Johnson, Blair Stevens, Ping Fang, and David Vo; Yale University School of Medicine, Department of Genetics, New Haven, Connecticut: Maurice J. Mahoney, Joanne R. Florio, and Peining Li; New Beginnings Perinatal Consultants: Providence, Rhode Island: Marshall Carpenter; University of Calgary, Early Assessment Risk Program, Calgary, Alberta, Canada: Jo-Ann Johnson and Melanie Pastuck; and University of Sydney, Perinatal Research Group, Kolling Institute of Medical Research, Sydney, New South Wales, Australia: Vitomir Tasevski and Jonathan Morris.

We are grateful to the staff of the Coordinating Center at Women \& Infants Hospital, Providence, Rhode Island, and in Standish, Maine_-specifically George J. Knight for assistance in protocol development and oversight, Regina Traficante for her role as Rhode Island study coordinator, Cheryl Felber for technical assistance, and Joanne Beaudoin for administrative assistance. We also thank Cindy Steinort (Electric Dreams, Scarborough, Maine) for developing the study-specific data-management system.

We also acknowledge the Sequenom Center for Molecular Medicine staff, including Roger Tim, Vivian Lu, John Tynan, Lesley Cagasan, Tricia Zwiefelhofer, Ron McCullough, Erin McCarthy, Anders Nygren, Jarrod Dean, Taylor Jensen, Frank Aquino, Sonia Espina-Grenie, Mengjia Tang, Julia Clemens, Wen Wu, Daniela Hubbard, Yabo Jin, Toni Paladino, Anna Maria Al-Khouri, Edlyn Mendoza, Paul Oeth , Kyle Ingersoll, Nick Miltgen, Anna Van Agtmael, Molly Dobb, Carlee Koessel, Shirin Fitzgerald, Lyle Rawlings, Melissa O'Day, Stephanie Lin, Haiping Lu, Rochelle Jean-Jacques, Vach Angkachatchai, Lin Tang, Tim Lu, Zhanyang Zhu, Zeljko Dzakula, Christine Chen, Tom Wang, Ryan Biltz, Benjamin Najara, Jennifer Wolanski, and David A. Henderson for the technical execution of the study.

Finally, we thank UCLA staff, including Zugen Chen for supervision of the next-generation resource, Tracy Toi for the project coordination and assay performance, Kingshuk Das for clinical interpretation, and Bret Harry for software implementation and processing of sample data.

\section{DISCLOSURE}

G.E.P. and J.A.C. were members of the Sequenom Clinical Advisory Board for 6 months and resigned when the study was funded. D.v.d.B., M.E., and A.T.B. are employees and shareholders of Sequenom, Inc. C.D. is an employee of Sequenom Center for Molecular Medicine and shareholder of Sequenom, Inc.

Sequenom, Inc., fully funded the project through a grant to Women \& Infants Hospital of Rhode Island. The Sequenom Center for Molecular Medicine (SCMM) was responsible for developing an internally validated laboratory-developed test for detecting Down syndrome in maternal plasma using massively parallel shotgun sequencing and for providing clinical interpretation of the test results. The SCMM also identified, equipped, and trained an independent laboratory to test a subset of samples through a separate contract with the University of California-Los Angeles (S.F.N. and W.W.G.). The sponsor did not control the study design, identify or communicate with enrollment sites, thaw or test samples before the formal testing period, have access to patient information before all testing was completed, analyze study results, prepare drafts of the manuscript, or have final decisions on manuscript content.

The other authors declare no conflict of interest.

\section{REFERENCES}

1. Savva GM, Walker K, Morris JK. The maternal age-specific live birth prevalence of trisomies 13 and 18 compared to trisomy 21 (Down syndrome). Prenat Diagn 2010;30:57-64

2. Data Access - Vital Statistics Online. Centers for Disease Control and Prevention http://www.cdc.gov/nchs/data_access/Vitalstatsonline.htm. Accessed November 2011.

3. Morris JK, Savva GM. The risk of fetal loss following a prenatal diagnosis of trisomy 13 or trisomy 18. Am J Med Genet A 2008;146:827-832.

4. Nembhard WN, Waller DK, Sever LE, Canfield MA. Patterns of first-year survival among infants with selected congenital anomalies in Texas, 19951997. Teratology 2001;64:267-275.

5. Rasmussen SA, Wong LY, Yang Q, May KM, Friedman JM. Population-based analyses of mortality in trisomy 13 and trisomy 18. Pediatrics 2003;111: 777-784 
6. Palomaki GE, Haddow JE, Knight GJ, et al. Risk-based prenatal screening for trisomy 18 using alpha-fetoprotein, unconjugated oestriol and human chorionic gonadotropin. Prenat Diagn 1995;15:713-723.

7. Palomaki GE, Neveux LM, Knight GJ, Haddow JE. Maternal serum-integrated screening for trisomy 18 using both first- and second-trimester markers. Prenat Diagn 2003;23:243-247.

8. Tul N, Spencer K, Noble P, Chan C, Nicolaides K. Screening for trisomy 18 by fetal nuchal translucency and maternal serum free beta-hCG and PAPP-A at 10-14 weeks of gestation. Prenat Diagn 1999;19: 1035-1042.

9. Ehrich M, Deciu C, Zwiefelhofer T, et al. Noninvasive detection of fetal trisomy 21 by sequencing of DNA in maternal blood: a study in a clinical setting. Am J Obstet Gynecol 2011;204:205.e1-205.11.

10. Palomaki GE, Kloza EM, Lambert-Messerlian GM, et al. DNA sequencing of maternal plasma to detect Down syndrome: an international clinical validation study. Genet Med 2011;13:913-920.

11. Chiu RW, Akolekar R, Zheng YW, et al. Non-invasive prenatal assessment of trisomy 21 by multiplexed maternal plasma DNA sequencing: large scale validity study. BMJ 2011;342:C7401.

12. Chiu RW, Chan KC, Gao Y, et al. Noninvasive prenatal diagnosis of fetal chromosomal aneuploidy by massively parallel genomic sequencing of DNA in maternal plasma. Proc Natl Acad Sci USA 2008;105: 20458-20463.

13. Fan HC, Blumenfeld YJ, Chitkara U, Hudgins L, Quake SR. Noninvasive diagnosis of fetal aneuploidy by shotgun sequencing DNA from maternal blood. Proc Natl Acad Sci USA 2008;105:16266-16271.

14. Chiu RW, Sun H, Akolekar R, et al. Maternal plasma DNA analysis with massively parallel sequencing by ligation for noninvasive prenatal diagnosis of trisomy 21. Clin Chem 2010;56:459-463.

15. Chen EZ, Chiu RW, Sun $\mathrm{H}$, et al. Noninvasive prenatal diagnosis of fetal trisomy 18 and trisomy 13 by maternal plasma DNA sequencing. PLOS ONE 2011;6:e21791.
16. Alkan C, Kidd JM, Marques-Bonet T, et al. Personalized copy number and segmental duplication maps using next-generation sequencing. Nat Genet 2009;41:1061-1067.

17. College of American Pathologists. Surveys 2011, FP-A Maternal Screening 2011

18. Wald NJ, Rodeck C, Hackshaw AK, Walters J, Chitty L, Mackinson AM. First and second trimester antenatal screening for Down's syndrome: the results of the Serum, Urine and Ultrasound Screening Study (SURUSS). J Med Screen 2003:10:56-104.

19. Nicolaides KH, Chervenak FA, McCullough LB, Avgidou K, Papageorghiou A. Evidence-based obstetric ethics and informed decision-making by pregnant women about invasive diagnosis after first-trimester assessment of risk for trisomy 21. Am J Obstet Gynecol 2005; 193:322-326.

20. Palomaki GE, Knight GJ, Haddow JE, Canick JA, Saller DN Jr, Panizza DS Prospective intervention trial of a screening protocol to identify fetal trisomy 18 using maternal serum alpha-fetoprotein, unconjugated oestriol, and human chorionic gonadotropin. Prenat Diagn 1992;12:925-930.

21. Haddow JE, Palomaki GE, Knight GJ, et al. Prenatal screening for Down's syndrome with use of maternal serum markers. N Engl J Med 1992;327:588-593.

22. ACOG. ACOG Practice Bulletin No. 88, December 2007. Invasive prenatal testing for aneuploidy. Obstet Gynecol 2007;110:1459-1467.

23. Tabor A, Philip J, Madsen M, Bang J, Obel EB, Nørgaard-Pedersen B. Randomised controlled trial of genetic amniocentesis in 4606 low-risk women. Lancet 1986:1:1287-1293.

(e) This work is licensed under the Creative Commons Attribution-Noncommercial-No Derivative Works 3.0 Unported License. To view a copy of this license, visit http://creativecommons.org/ licenses/by-nc-nd/3.0/ 